\title{
Caracterização das quedas em idosos socorridos pelo serviço de atendimento móvel de urgência
}

\section{Characterization of falls among elderly rescued by the mobile first-aid service}

Kennia Sibelly Marques de Abrantes ${ }^{1,2}$, Tarciana Nobre de Menezes ${ }^{3}$, Maria do Carmo Andrade Duarte de Farias 2,4 ,

Maria Isabel Leandro da Silva ${ }^{1}$, Vanessa Estrela Rolim¹, Hugo Macedo Junior ${ }^{2}$, Luiz Carlos de Abreu²

${ }^{1}$ Curso de Enfermagem da Universidade Federal de Campina Grande (UFCG) - Cajazeiras (PB), Brasil.

${ }^{2}$ Laboratório de Escrita Científica e Delineamento de Estudos do Departamento de Morfologia e Fisiologia da Faculdade de Medicina do ABC (FMABC) - Santo André (SP), Brasil.

${ }^{3}$ Departamento de Fisioterapia e Programa de Pós-Graduação em Saúde Pública da Universidade Estadual da Paraíba (UEPB) - Campina Grande (PB), Brasil.

${ }^{4}$ Escola Técnica de Saúde de Cajazeiras da UFCG - Cajazeiras (PB), Brasil.

DOI: http://dx.doi.org/10.7322/abcshs.v38i3.17

\section{RESUMO}

Introdução: Atualmente, o aumento da expectativa de vida tem chamado a atenção quanto às condições de saúde durante esses anos adicionais e quanto à possibilidade de incidências futuras de morbidades e mortalidade e diversos problemas de saúde, como as quedas. Objetivo: Verificar a associação entre as características da queda e o grupo etário. Métodos: Trata-se de um estudo transversal. Os dados foram coletados por meio de um formulário específico nos meses de março a junho de 2012 e analisados por meio do Statistical Package for the Social Sciences (SPSS, versão 17.0). As associações foram verificadas por meio do teste $\chi^{2}$ de Pearson ou do teste exato de Fisher com extensão de Freeman-Halton, quando apropriado, sendo adotado o nível de significância $\alpha<5 \%$. Resultados: Observou-se um total de 93 idosos vítimas de quedas. Prevaleceu o grupo de faixa etária igual ou maior que 80 anos (47,3\%), do sexo feminino $(62,4 \%)$, com ensino fundamental incompleto ou acima (51,6\%), sem renda ou com renda de até um salário mínimo (66,7\%), residindo sem companheiro $(65,6 \%)$. A ocorrência de quedas em idosos mostrou associação entre grupo etário e sexo $(p=0,004)$, situação conjugal $(p=0,002)$, hospitalização $(p=0,047)$ e lugar da queda $(p=0,006)$. Conclusão: $O s$ idosos com 70 anos ou mais foram os que se apresentaram em maior proporção no tempo de hospitalização, e, no que se refere ao lugar de ocorrência da queda, observou-se que, entre os idosos mais velhos, destacou-se um maior percentual de quedas em casa, principalmente quando comparados aos idosos mais jovens.

Palavras-chave: acidentes por quedas; idoso; serviços médicos de emergência; socorro de urgência

\begin{abstract}
Introduction: Currently the increase in life expectancy has called attention to the health conditions during those extra years and the possibility of future incidences of morbidity and mortality and various health problems such as falls. Objective: To investigate the association between the characteristics of fall and age group. Methods: This was a cross-sectional study. Data were collected through a specific form in the months from March to June 2012 and analyzed using the Statistical Package for Social Sciences (SPSS, version 17.0). The associations were assessed using the $\chi^{2}$ test or Fisher's exact test with Freeman-Halton extension, where appropriate, adopting a significance level $\alpha<5 \%$. Results: A total of 93 elderly victims of falls. The most prevalent group was of age equal to or greater than 80 years (47.3\%), female $(62.4 \%)$, with incomplete primary education or above (51.6\%), no income or income below the poverty level (66.7\%), living without a partner (65.6\%). The occurrence of falls in the elderly showed an association between age group and sex $(p=0.004)$, marital status $(p=0.002)$, hospitalization $(p=0.047)$ and place of fall $(p=0.006)$. Conclusion: Elderly patients 70 years or older were those who presented in greater proportion to length of stay and, in relation to the place of occurrence of the fall, it was observed that, among the oldest old, stood out a higher percentage falls at home, especially when compared to younger participants.
\end{abstract}

Keywords: accidental falls; aged; emergency medical services; emergency relief. 


\section{INTRODUÇÃO}

Atualmente, o aumento da expectativa de vida representa um fenômeno mundial, que resulta no envelhecimento populacional, o que requer profundas mudanças para as políticas sociais, configurando-se como um dos maiores desafios da saúde pública ${ }^{1}$.

Esse fenômeno tem chamado a atenção quanto às condições de saúde durante esses anos adicionais de vida e quanto à possibilidade de incidências futuras de morbidades e mortalidade, visto que, com o envelhecimento, surgem diversos problemas de saúde, decorrentes das "perdas" funcionais do processo fisiológico, que comprometem as funções do organismo e provocam alterações no estilo de vida dos idosos².

Assim, com o constante crescimento da população de idosos, vê-se a necessidade de adequado conhecimento sobre os principais problemas que os atingem, dentre os quais se destacam as quedas $^{3,4}$. Entende-se que queda é o deslocamento não intencional do corpo para um nível inferior à posição inicial, com incapacidade de correção de tempo hábil, determinado por circunstâncias multifatoriais, comprometendo a estabilidade 3 .

As quedas representam grave problema de saúde pública, por ser um agravo frequente e limitante, cujo impacto tem sido despercebido pela sociedade brasileira, por considerarem que, com o envelhecimento, estas são inevitáveis. Contudo, são eventos mórbidos, causadores de lesão, de distúrbios emocionais, de declínio funcional e morte, que deveriam ser prevenidos ${ }^{4}$.

Quanto aos aspectos epidemiológicos, estima-se que um terço dos idosos que vivem na comunidade sofrerá queda no intervalo de um ano e, entre os institucionalizados, essa previsão aumenta para $50 \%{ }^{5}$.

No Brasil, aproximadamente $30 \%$ dos idosos caem uma vez por ano e o risco de cair aumenta, significativamente, com o avançar da idade. A estimativa de quedas, por faixa etária, é de $32 \%$ entre os 65 e 74 anos, $35 \%$ entre os 75 e os 84 anos e $51 \%$, acima dos 85 anos $^{6,7}$. Em 2005, as quedas foram responsáveis por $54 \%$ das causas externas de internações hospitalares em indivíduos com 60 anos ou mais e por $70 \%$ das mortes acidentais de pessoas com 75 anos e mais ${ }^{4,8}$.

A origem da queda pode estar associada a fatores intrínsecos, decorrentes de alterações fisiológicas do envelhecimento, deterioração cognitiva, redução da capacidade funcional, condições patológicas e efeitos adversos de medicação, e a fatores extrínsecos, destacando-se as circunstâncias sociais e os perigos ambientais que oferecem desafios ao idoso. Mediante esses fatores, torna-se difícil restringir um evento de queda a um único fator de risco ou a um agente causal ${ }^{9}$.

Pessoas de todas as idades podem sofrer quedas, porém, nos idosos, ela apresenta um significado relevante, uma vez que podem levá-los a incapacidade, injúria e morte. Esse evento causa impacto na saúde e tem custos sociais e econômicos imensos relacionados a diagnóstico, tratamento e reabilitação que se acentuam quando o idoso tem diminuição da autonomia e da independência ${ }^{10}$.
Assim, o objetivo é verificar a associação entre as características da queda e o grupo etário.

\section{MÉTODOS}

Este estudo de corte transversal foi realizado no Serviço de Atendimento Móvel de Urgência (SAMU) localizado no município de Sousa (PB). Esse município está situado no alto sertão paraibano, apresenta área territorial de $842.000 \mathrm{~km}^{2}$, e população estimada em 65.807 mil habitantes de acordo com dados do Instituto Brasileiro de Geografia e Estatística (IBGE) ${ }^{11}$ do ano de 2010.

O SAMU é um serviço avançado que se fundamenta na Política Nacional de Atenção às Urgências e tem por finalidade prestar socorro à população em casos de urgência e/ou emergência.

A amostra foi constituída por todos os idosos traumatizados socorridos pelo SAMU, no período de 01 de janeiro a 31 de dezembro de 2011, cujo trauma foi em decorrência de queda. Foram atendidos, nesse período, 124 idosos.

Para realização desta pesquisa, considerou-se o conceito de idoso da Organização das Nações Unidas (ONU), a qual determina que, para os países desenvolvidos, indivíduos com idade a partir de 65 anos são considerados idosos e, para os países em desenvolvimento, onde a expectativa média de vida é menor, adotam-se os 60 anos como a idade de transição das pessoas para o segmento idoso da população ${ }^{12}$.

A coleta de dados foi realizada no período de janeiro a maio de 2012 e consistiu, no primeiro momento, em identificar nome, endereço e idade dos idosos a partir não só das fichas de ocorrência, preenchidas durante os atendimentos, como também das fichas preenchidas pelos Técnicos de Regulação Médica (TARM).

No segundo momento, os idosos foram localizados e entrevistados em seus domicílios, mediante a anuência pela assinatura do Termo de Consentimento Livre e Esclarecido (TCLE). Nessa ocasião, responderam às perguntas relativas aos dados sociodemográficos (sexo, situação conjugal, grau de escolaridade, profissão, renda e com quem mora), às características das quedas (tipo de queda - da própria altura, da cama, problemas com degrau), à hospitalização (não, sim - até 12 horas ou 13 horas ou mais), às sequelas (não, sim - deambula com dificuldade, não deambula, perda da função de um membro e/ou órgão), lugar (próprio domicílio ou via pública) e causa da queda (tontura/desequilíbrio, fatores ambientais, embriaguez) e ainda consequências das quedas (medo de voltar a cair, abandono de atividades, imobilização, ajuda para realizar atividades, alterações no rearranjo familiar, mudança de domicílio, modificação de hábitos, isolamento e depressão). As informações sobre as consequências foram múltiplas.

Quando necessário, a entrevista com o idoso foi realizada na presença de um familiar ou cuidador, que confirmou e/ou esclareceu as informações obtidas. Na impossibilidade de o idoso responder ao questionário ou quando, em decorrência da queda, 
evoluiu para o óbito, este foi respondido por um familiar ou cuidador. Da amostra estudada, 11 idosos evoluíram para o óbito.

Os dados foram analisados por meio do aplicativo estatístico Statistical Package for the Social Sciences (SPSS), versão 17.0 for Windows. As associações entre os dados sociodemográficos e as características das quedas com o grupo etário foram verificadas por meio do teste $\chi^{2}$ de Pearson, ou teste exato de Fisher com extensão de Freeman-Halton, quando apropriado, sendo adotado o nível de significância $\alpha<5 \%$. A pesquisa foi aprovada pelo Comitê de Ética em Pesquisa (CEP) da UEPB sob o número de protocolo (CAAE 0763.0.133.000-11).

\section{RESULTADOS}

Das 124 vítimas, não foi possível a localização do endereço de 24 idosos, seis se recusaram a participar da pesquisa, e um encontrava-se viajando durante o período da coleta. Dessa forma, entre perdas e recusas (31), foram avaliados 93 idosos.

Do total de idosos entrevistados, $62,4 \%$ eram constituídos por idosos do sexo feminino, $65,6 \%$ não viviam com companheiro, $51,6 \%$ tinham ensino fundamental incompleto ou acima, $66,7 \%$ não tinham renda ou ganhavam até um salário-mínimo. A faixa etária mais prevalente foi de 80 anos ou mais (47,3\%), seguida de 70 a 79 anos $(33,3 \%)$ e 60 a 69 anos $(19,4 \%)$. A idade dos idosos variou de 60 a 104 anos, com média de 79 anos ( $D P=10,5$ anos) e mediana de 78 anos.

Na Tabela 1 é apresentada a distribuição dos idosos estudados de acordo com as variáveis sociodemográficas e o grupo etário. É possível observar que a maior proporção de idosos de 60 a 69 anos era constituída por homens $(61,1 \%)$ e a maior parte dos idosos com 80 anos ou mais era de mulheres (79,5\%). Foi observada associação estatisticamente significativa entre sexo e grupo etário ( $\mathrm{p}=0,004)$.

Nessa tabela observa-se ainda, com relação à situação conjugal, que a maioria dos idosos com 80 anos ou mais e com 70 a 79 anos vivia sem companheiro $(84,1$ e $51,6 \%$, respectivamente). Entre os idosos com 60 a 69 anos sobressairam-se aqueles que viviam com companheiro $(55,6 \%)$. Foi observada associação estatisticamente significativa entre situação conjugal e grupo etário $(\mathrm{p}=0,002)$.

Quanto à escolaridade, observa-se que os idosos com 80 anos ou mais apresentaram elevada proporção de analfabetismo (56,8\%), quando comparados aos demais grupos etários. Nos grupos etários de 60 a 69 anos e 70 a 79 anos, a maioria foi de idosos que referiram ter ensino fundamental incompleto ou acima deste (55,6 e 61,3\%, respectivamente). Não foi observada associação entre escolaridade e grupo etário ( $\mathrm{p}=0,283)$.

Quanto à renda, a maioria dos idosos de todos os grupos etários apresentava renda de 0 a 1 salário-mínimo. Não foi encontrada associação estatisticamente significativa entre renda e grupo etário $(\mathrm{p}=0,119)$ (Tabela 1).

$\mathrm{Na}$ Tabela 2 são apresentadas as informações sobre a distribuição dos idosos estudados de acordo com as características das quedas e grupo etário.

Os dados da Tabela 2 mostram que, entre os idosos dos três grupos etários, prevaleceram as quedas da própria altura, sendo $79,5 \%$ entre os idosos com 80 anos ou mais, $74,2 \%$ entre os com idade entre 70 e 79 anos e $61,1 \%$ entre os com idade entre 60 e 69 anos. Não foi observada associação estatisticamente significativa entre o tipo de queda e o grupo etário $(\mathrm{p}=0,322)$.

Quanto à necessidade de hospitalização, entre os idosos de 60 a 69 anos, a maior parte não precisou de hospitalização $(38,9 \%)$, assim como entre os idosos com 80 anos mais (50,0\%). Entre os idosos com 70 a 79 anos, 58,1\% precisaram ficar hospitalizados por 13 horas ou mais. Foi verificada associação estatisticamente significativa entre hospitalização e grupo etário $(\mathrm{p}=0,047)$.

Quando indagados sobre o surgimento de sequelas em decorrência da queda, $53,8 \%$ dos idosos com 80 anos ou mais referiram a sua presença. Entre os que não apresentaram destacam-se aqueles entre 70 e 79 anos $(35,8 \%)$ e 60 e 69 anos (19,4\%). Esses dados demonstram maior frequência de sequelas entre os idosos mais velhos. Não foi verificada associação estatisticamente significativa entre sequelas e grupo etário $(\mathrm{p}=0,680)$.

A respeito do lugar onde ocorreu a queda, 57,6\% dos idosos do grupo de 80 anos ou mais referiram ter acontecido em casa. Nos grupos de 70 a 79 anos $(44,4 \%)$ e 60 a 69 anos $(33,3 \%)$,

Tabela 1: Distribuição dos idosos estudados, de acordo com as informações sociodemográficas e grupo etário - Sousa (PB), 2011

\begin{tabular}{|c|c|c|c|c|c|c|c|}
\hline \multirow{3}{*}{ Variáveis sociodemográficas } & \multicolumn{6}{|c|}{ Grupo etário } & \multirow{3}{*}{ valor $p$} \\
\hline & \multicolumn{2}{|c|}{60 a 69 anos } & \multicolumn{2}{|c|}{70 a 79 anos } & \multicolumn{2}{|c|}{80 anos ou mais } & \\
\hline & $\mathbf{n}$ & $\%$ & $\mathbf{n}$ & $\%$ & $\mathbf{n}$ & $\%$ & \\
\hline $\begin{array}{l}\text { Sexo } \\
\text { Feminino } \\
\text { Masculino }\end{array}$ & $\begin{array}{c}7 \\
11\end{array}$ & $\begin{array}{l}38,9 \\
61,1\end{array}$ & $\begin{array}{l}16 \\
15\end{array}$ & $\begin{array}{l}51,6 \\
48,4\end{array}$ & $\begin{array}{c}35 \\
9\end{array}$ & $\begin{array}{l}79,5 \\
20,5\end{array}$ & 0,004 \\
\hline $\begin{array}{l}\text { Situação conjugal } \\
\text { Com companheiro } \\
\text { Sem companheiro }\end{array}$ & $\begin{array}{c}10 \\
8\end{array}$ & $\begin{array}{l}55,6 \\
44,4\end{array}$ & $\begin{array}{l}15 \\
16\end{array}$ & $\begin{array}{l}48,4 \\
51,6\end{array}$ & $\begin{array}{c}7 \\
37\end{array}$ & $\begin{array}{l}15,9 \\
84,1\end{array}$ & 0,002 \\
\hline $\begin{array}{l}\text { Escolaridade } \\
\text { Analfabeto } \\
\text { Ensino fundamental incompleto ou acima }\end{array}$ & $\begin{array}{c}8 \\
10\end{array}$ & $\begin{array}{l}44,4 \\
55,6\end{array}$ & $\begin{array}{l}12 \\
19\end{array}$ & $\begin{array}{l}38,7 \\
61,3\end{array}$ & $\begin{array}{l}25 \\
19\end{array}$ & $\begin{array}{l}56,8 \\
43,2\end{array}$ & 0,283 \\
\hline $\begin{array}{l}\text { Renda em salários-mínimos } \\
0 \text { a } 1 \text { salário-mínimo } \\
2 \text { ou mais salários-mínimos }\end{array}$ & $\begin{array}{r}10 \\
8\end{array}$ & $\begin{array}{l}55,6 \\
44,4\end{array}$ & $\begin{array}{l}18 \\
13\end{array}$ & $\begin{array}{l}58,1 \\
41,9\end{array}$ & $\begin{array}{l}34 \\
10\end{array}$ & $\begin{array}{l}77,3 \\
22,7\end{array}$ & 0,119 \\
\hline
\end{tabular}


Tabela 2: Distribuição dos idosos estudados de acordo com as características da queda e grupo etário - Sousa (PB), 2011

\begin{tabular}{|c|c|c|c|c|c|c|c|}
\hline \multirow{3}{*}{ Categorias } & \multicolumn{6}{|c|}{ Grupo etário } & \multirow{3}{*}{ valor $p$} \\
\hline & \multicolumn{2}{|c|}{60 a 69 anos } & \multicolumn{2}{|c|}{70 a 79 anos } & \multicolumn{2}{|c|}{80 anos ou mais } & \\
\hline & $\mathbf{n}$ & $\%$ & n & $\%$ & $n$ & $\%$ & \\
\hline $\begin{array}{l}\text { Tipo de queda } \\
\text { Da própria altura } \\
\text { Outros }\end{array}$ & $\begin{array}{l}11 \\
7\end{array}$ & $\begin{array}{l}61,1 \\
38,9\end{array}$ & $\begin{array}{c}23 \\
8\end{array}$ & $\begin{array}{l}74,2 \\
25,8\end{array}$ & $\begin{array}{c}35 \\
9\end{array}$ & $\begin{array}{l}79,5 \\
20,5\end{array}$ & 0,322 \\
\hline $\begin{array}{l}\text { Hospitalização } \\
\text { Até } 12 \text { horas } \\
13 \text { horas ou mais } \\
\text { Não }\end{array}$ & $\begin{array}{l}6 \\
5 \\
7\end{array}$ & $\begin{array}{l}33,3 \\
27,8 \\
38,9\end{array}$ & $\begin{array}{c}6 \\
18 \\
7\end{array}$ & $\begin{array}{l}19,4 \\
58,1 \\
22,6\end{array}$ & $\begin{array}{l}10 \\
12 \\
22\end{array}$ & $\begin{array}{l}22,7 \\
27,3 \\
50,0\end{array}$ & 0,047 \\
\hline $\begin{array}{l}\text { Sequelas } \\
\text { Presente } \\
\text { Ausente }\end{array}$ & $\begin{array}{c}5 \\
13\end{array}$ & $\begin{array}{l}27,8 \\
72,2\end{array}$ & $\begin{array}{c}7 \\
24\end{array}$ & $\begin{array}{l}22,6 \\
77,4\end{array}$ & $\begin{array}{l}14 \\
30\end{array}$ & $\begin{array}{l}31,8 \\
68,2\end{array}$ & 0,680 \\
\hline $\begin{array}{l}\text { Lugar da queda } \\
\text { Em casa } \\
\text { Na rua }\end{array}$ & $\begin{array}{l}9 \\
9\end{array}$ & $\begin{array}{l}50,0 \\
50,0\end{array}$ & $\begin{array}{l}19 \\
12\end{array}$ & $\begin{array}{l}61,3 \\
38,7\end{array}$ & $\begin{array}{c}38 \\
6\end{array}$ & $\begin{array}{l}86,4 \\
13,6\end{array}$ & 0,006 \\
\hline $\begin{array}{l}\text { Causa da queda* } \\
\text { Tontura/desequilíbrio } \\
\text { Fatores ambientais } \\
\text { Embriaguez }\end{array}$ & $\begin{array}{l}7 \\
9 \\
2\end{array}$ & $\begin{array}{l}38,9 \\
50,0 \\
11,1\end{array}$ & $\begin{array}{c}14 \\
17 \\
0\end{array}$ & $\begin{array}{c}45,2 \\
54,8 \\
0,0\end{array}$ & $\begin{array}{c}25 \\
19 \\
0\end{array}$ & $\begin{array}{c}56,8 \\
43,2 \\
0,0\end{array}$ & 0,113 \\
\hline
\end{tabular}

*Realizado teste exato de Fisher com extensão de Freeman-Halton

Tabela 3: Distribuição dos idosos estudados de acordo com as consequências das quedas para sua vida - Sousa (PB), 2011

\begin{tabular}{|l|c|c|}
\hline Consequências da queda* & n & $\%$ \\
\hline Medo de voltar a cair & 60 & 64,5 \\
\hline Necessida de ajuda em atividades & 50 & 53,8 \\
\hline Abandono de atividades & 45 & 48,4 \\
\hline Modificação de hábitos & 42 & 45,2 \\
\hline Imobilização & 25 & 26,9 \\
\hline Isolamento & 15 & 16,1 \\
\hline Rearranjo familiar & 13 & 14,0 \\
\hline Mudança de domicílio & 5 & 5,4 \\
\hline Depressão & 2 & 2,2 \\
\hline
\end{tabular}

${ }^{*}$ Resposta múltipla

destacou-se a rua como principal lugar para a ocorrência de quedas. Foi verificada associação estatisticamente significativa entre lugar da queda e grupo etário $(\mathrm{p}=0,006)$.

Quando questionados sobre a causa atribuída à queda, destacou-se, entre os idosos mais velhos (80 anos ou mais), tontura/ desequilíbrio (54,3\%), assim como os fatores ambientais (42,2\%). Nos grupos de 70 a 79 anos e 60 a 69 anos, sobressairam-se os fatores ambientais com $37,8 \%$ e $20,0 \%$, respectivamente. Não foi verificada associação estatisticamente significativa entre causa da queda e grupo etário ( $\mathrm{p}=0,113$ ) (Tabela 2 ).

As consequências das quedas relatadas pelos idosos são apresentadas na Tabela 3, sendo possível observar que $64,5 \%$ dos idosos referiram o medo de voltar a cair, seguido pela necessidade de ajuda para realizar atividades $(53,8 \%)$, abandono de atividade $(48,4 \%)$ e modificação de hábitos $(45,2 \%)$.

\section{DISCUSSÃO}

As quedas representam um grave problema de saúde pública, constituindo a principal etiologia de morte acidental em pessoas com idade acima de 65 anos e pode levar a incapacidade, injúria ou morte ${ }^{13}$. A maioria das quedas resulta da interação de fatores relacionados ao indivíduo (intrínsecos), que são decorrentes de alterações fisiológicas do envelhecimento, e fatores ambientais (extrínsecos), que são as circunstâncias sociais e ambientais que desafiam o idoso ${ }^{4,9}$. Diante disso, os diferentes aspectos relacionados à queda têm sido temática constante em pesquisas realizadas em diferentes países ${ }^{14-17}$, como é o caso do Brasil ${ }^{18-20}$.

Neste estudo foi verificada predominância de idosos do sexo feminino (62,4\%). Resultado semelhante foi encontrado em outras pesquisas realizadas com idosos ${ }^{21,22}$, assim como entre os idosos acometidos por quedas ${ }^{23-25}$. Em estudo realizado em um município do Rio de Janeiro, com idosos acometidos por quedas, também foi verificada maior proporção de mulheres entre os indivíduos estudados ${ }^{26}$.

O processo de envelhecimento ocasiona mudanças estruturais e funcionais que podem comprometer as funções motoras, impedir a capacidade de adaptação do indivíduo ao ambiente e predispô-lo a ocorrência de quedas. No caso específico das mulheres, além dos fatores já citados, pode ocorrer maior perda de massa óssea devido à redução do estrógeno, adicionando riscos para o surgimento da osteoporose, maior exposição a atividades domésticas, menor massa magra e força muscular e maior prevalência de doenças crônicas ${ }^{6}$. No entanto, embora vivam mais que os homens, passam por um período maior de debilitação física que gera comprometimento de sua saúde, o que provavelmente explica a maior incidência de quedas ${ }^{27}$.

Neste estudo não foi verificada associação estatisticamente significativa entre escolaridade, renda e grupo etário.

Importantes avanços nos níveis educacionais da população brasileira ocorreram, mas a escolaridade dos idosos é ainda considerada baixa, 30,7\% tinham menos de um ano de instrução em 2010. Em 2009, 50,2\% tinham menos de quatro anos de estudo, $32,3 \%$ de quatro a oito anos e $17,4 \%$ tinham nove anos ou mais de estudo ${ }^{28}$.

Porém, é importante destacar que o Brasil alcançou nas últimas décadas um crescimento considerável quanto ao acesso à escola. Nas áreas rurais, por exemplo, onde a oferta de 
estabelecimentos que visam à educação é mais reduzida, o crescimento foi bastante significativo, passando de $15,2 \%$ para $28,4 \%{ }^{28}$. Assim, pode-se deduzir que em alguns anos os níveis de escolaridade e socioeconômico terão melhorado, o que influenciará nos que se tornarão idosos.

Neste estudo foi verificada associação significativa entre sexo e grupo etário, em que maior proporção de idosos com 80 anos ou mais é do sexo feminino e com 60 a 69 anos é do sexo masculino. Estudos com idosos têm verificado resultados semelhantes, nos quais a maior proporção de idosos mais velhos é constituída por mulheres ${ }^{29-31}$.

Enquanto as mulheres vivem mais, principalmente, por estarem menos expostas a acidentes de trabalho, ao consumo de álcool, ao tabagismo, à morte por causas externas e doenças cardiovasculares $^{32}$, os homens morrem mais precocemente, pois demoram a buscar os serviços de saúde e não aderem aos tratamentos, principalmente os de longa duração ${ }^{33}$.

Com relação à situação conjugal, maior proporção de idosos com 60 a 69 anos vivia com o companheiro e maior proporção de idosos com 80 anos ou mais não vivia com companheiro.

A ocorrência de quedas entre os idosos que não têm união estável pode estar relacionada ao fato de não ter alguém para compartilhar cooperação mútua nos cuidados com a saúde, hábito considerado comum entre os casais ${ }^{34}$. Além disso, entre as consequências da queda, tem se observado que idosos com menor convívio com pessoas apresentam maior probabilidade de medo de novas quedas, o que pode ocasionar restrição de atividades e diminuição na autoconfiança, interferindo diretamente na qualidade de vida ${ }^{35}$.

Neste estudo não foi observada associação significativa entre tipo de queda e sequelas com o grupo etário. No entanto, há que se considerar a elevada proporção de idosos que caíram da própria altura, principalmente entre os mais velhos. A idade avançada constitui fator de risco para o evento, uma vez que o envelhecimento provoca perda de equilíbrio, alterações na massa muscular e óssea e maior restrição na atividade física, aumentando a ocorrência de quedas ${ }^{25}$.

De forma semelhante ao que ocorreu na presente pesquisa, foi verificado, em outro estudo ${ }^{36}$, que, à medida que a idade evoluiu, aumentou o número de quedas da própria altura, principalmente na faixa etária acima de 81 anos (85,7\%).

A queda da própria altura pode estar relacionada a problemas com o ambiente, como piso escorregadio, atrapalhar-se com objetos no chão, trombar em outras pessoas, subir em objetos para alcançar algo, queda da cama, problemas com degrau e outros ${ }^{10}$.

As condições de moradia dos idosos entrevistados sinalizavam um ambiente inseguro, devido à presença de obstáculos, tais como degraus, piso escorregadio, tapete solto e pouca iluminação, que podem ser considerados como fatores predisponentes às quedas. Esses obstáculos variavam de acordo com as estruturas domiciliares, com os cômodos mais utilizados e com os hábitos individuais de cada idoso.
Em todos os grupos etários verificou-se elevada proporção de idosos que precisaram de hospitalização. Os idosos com 70 anos ou mais foram os que se apresentaram em maior proporção com tempo de hospitalização de 13 horas ou mais.

As quedas são o principal tipo de agravo que resulta em hospitalização de idosos, a esse respeito, foi verificado, em um estudo com idosos traumatizados, que $36,5 \%$ permaneceram internados no período de um a dez dias, $31,7 \%$ permaneceram de 11 a 20 dias e $20,9 \%$ ficaram hospitalizados no período de 30 a 120 dias $^{37}$.

Uma pesquisa sobre a mortalidade de idosos que sofreram fraturas graves revelou que o risco de morte dentro do período de um ano após a ocorrência da fratura em decorrência de queda foi cerca de seis vezes maior entre os indivíduos admitidos para internação ${ }^{38}$.

No que se refere ao lugar de ocorrência das quedas, observou-se que, entre os idosos mais velhos, destacou-se um maior percentual de quedas em casa, principalmente quando comparados aos idosos mais jovens. A maior parte das quedas ocorrendo no próprio domicílio também foi encontrado em outros estudos ${ }^{9,25}$.

As condições ambientais de moradia com a presença de obstáculos, tais como degraus, piso escorregadio, tapete solto e pouca iluminação são fatores predisponentes às quedas e variam de acordo com as estruturas domiciliares, com os cômodos mais utilizados e com os hábitos individuais de cada idoso, o que justifica a implantação de medidas preventivas em função da estreita relação entre ambiente e quedas ${ }^{19}$.

Neste estudo, não foi observada associação significativa entre causa atribuída à queda e grupo etário, no entanto, observou-se que os fatores relacionados ao indivíduo e ao ambiente estiveram mais presentes com a idade aumentada, uma vez que nos idosos mais velhos foi elevada a proporção de tontura/desequilíbrio e de fatores ambientais.

As causas das quedas em idosos podem ser variadas e estarem associadas, resultando da interação de fatores relacionados ao indivíduo (intrínsecos), que envolvem alterações fisiológicas do envelhecimento, idade avançada, doenças existentes e fatores ambientais (extrínsecos), que se referem às condições que oferecem riscos no ambiente ${ }^{4}$.

Em relação às consequências das quedas, no presente estudo destacaram-se principalmente medo de voltar a cair, necessidade de ajuda para realizar atividades, abandono de atividades, modificação de hábitos e imobilização.

O medo de voltar a cair destaca-se entre as demais consequências e é revestido por sentimentos de fragilidade e insegurança, pois ocasiona alterações como perda de autonomia e independência para as atividades sociais e da vida diária. No entanto, pode também ser encarado como um fator protetor, na medida em que o idoso se conscientiza e adota comportamentos preventivos ${ }^{9}$.

As quedas podem gerar diversas consequências na vida de um idoso, incluindo morbidade, mortalidade, declínio funcional, 
hospitalização, institucionalização e elevado custo social e econômico decorrentes das lesões provocadas, configurando-se como um importante problema de saúde pública ${ }^{6}$.

Houve associação entre hospitalização e lugar da queda e grupo etário. Isso porque, quanto à hospitalização, os idosos com 70 anos ou mais foram os que se apresentaram em maior proporção com tempo de hospitalização e, no que se refere ao lugar de ocorrência das quedas, observou-se, entre os idosos mais velhos (80 anos ou mais), que houve um maior percentual de quedas em casa.

Dessa forma, é necessário que os profissionais de saúde atuem de forma que ofereçam aos idosos uma assistência à saúde que priorize aspectos da promoção do envelhecimento saudável e com significativa capacidade funcional, para, assim, proporcionar meios que previnam a ocorrência de quedas ${ }^{39}$.

\section{REFERÊNCIAS}

1. Garbin CAS, Sumida DH, Moimaz SAS, Prado RL, Silva MM. O envelhecimento na perspectiva do cuidador de idosos. Ciênc Saúde Coletiva. 2010;15(6):2941-8.

2. Parahyba MI, Veras R. Diferenciais sociodemográficos no declínio funcional em mobilidade física entre os idosos no Brasil. Ciênc Saúde Coletiva. 2008;13(4):1257-64.

3. Alvares LM, Lima RC, Silva RA. Ocorrência de quedas em idosos residentes em instituições de longa permanência em Pelotas, Rio Grande do Sul, Brasil. Cad Saúde Pública. 2010;26(1):31-40.

4. Maciel A. Quedas em idosos: um problema de saúde pública desconhecido pela comunidade e negligenciado por muitos profissionais da saúde e por autoridades sanitárias brasileiras. Rev Méd Minas Gerais. 2010;20(4):554-7.

5. Nicolussi AC, Fhon JRS, Santos CAV, Kusumota L, Marques $S$, Rodrigues RAP. Qualidade de vida em idosos que sofreram quedas: revisão integrativa da literatura. Ciênc Saúde Coletiva. 2012;17(3):723-30

6. Cruz DT, Ribeiro LC, Vieira MT, Teixeira MTB, Bastos RR, Leite ICG. Prevalência de quedas e fatores associados em idosos. Rev Saúde Pública. 2012;46(1):138-46.

7. Silva A, Almeida GJM, Cassilhas RC, Cohen M, Peccin MS, Tufik $\mathrm{S}$, et al. Equilíbrio, coordenação e agilidade de idosos submetidos à prática de exercícios físicos resistidos. Rev Bras Med Esporte. 2008;14(2):88-93.

8. Ministério da Saúde [Internet]. Sistema de Informações hospitalares do SUS (SIH/SUS). Disponível em: http://tabnet.datasus.gov.br/cgi/ idb2007/matriz.htm\#demog. Acesso em: 1 fev. 2013.

9. Ribeiro AP, Souza ER, Atie S, Souza AC, Schilithz AO. A influência das quedas na qualidade de vida de idosos. Ciênc Saúde Coletiva. 2008;13(4):1265-73.

10. Fabrício SCC, Rodrigues RAP, Costa Junior ML. Causas e consequências de quedas de idosos atendidos em hospital público. Rev Saúde Pública. 2004;38(1):93-9.

11. Instituto Brasileiro de Geografia e Estatística [Internet]. Disponível em: http://cidades.ibge.gov.br. Acesso em: 26 set. 2013.

12. Paschoal SMP, Salles RFN, Franco RP. Epidemiologia do Envelhecimento. In: Carvalho Filho ET, Papaléo Netto M, editors. Geriatria: Fundamentos, clínica e terapêutica. São Paulo: Atheneu; 2005. cap. 4. p. 39-56.

13. Calil AM, Paranhos WY. O Enfermeiro e as situações de emergência. São Paulo: Atheneu; 2007.

14. Lim YM, Sung MH. Home environmental and health-related factors among home fallers and recurrent fallers in community dwelling older Korean women. Int J Nurs Pract. 2012;18(5):481-8.

15. Limpawattana P, Sutra S, Thavompitak Y, Chindaprasirt J, Mairieng P. Geriatric hospitalizations due to fall-related injuries. J Med Assoc Thai. 2012;95(7):235-9.
16. Sterke CS, Van Beeck EF, Van Der Velde N, Ziere G, Petrovic $\mathrm{M}$, Looman CW, et al. New insights: dose-response relationship between psychotropic drugs and falls: a study in nursing home residents with dementia. J Clin Pharmacol. 2012;56(6):947-55.

17. Gnjidic D, Hilmer SN, Blyth FM, Naganathan V, Waite L, Seibel $\mathrm{MJ}$, et al. Polypharmacy cutoff and outcomes: five or more medicines were used to identify community-dwelling older men at risk of different adverse outcomes. J Clin Epidemiol. 2012;65(9):989-95

18. Beck AP, Antes DL, Meurer ST, Benedetti TRB, Lopes MA. Fatores associados às quedas entre idosos praticantes de atividades físicas. Texto Contexto Enferm. 2011;20(2):280-6.

19. Ferreira DCO, Yoshitome AY. Prevalência e características das quedas de idosos institucionalizados. Rev Bras Enferm. 2010;63(6):991-7

20. Menezes RL, Bachion MM. Estudo da presença de fatores de riscos intrínsecos para quedas, em idosos institucionalizados. Ciênc Saúde Coletiva. 2008;13(4):1209-18.

21. Cardoso JH, Costa JSD. Características epidemiológicas, capacidade funcional e fatores associados em idosos de um plano de saúde. Ciênc Saúde Coletiva. 2010;15(6):2871-8.

22. Campos JFS, Poletti NAA, Rodrigues CDS, Garcia TPR, Angelini JF, Von Dollinger APA, et al. Trauma em idosos atendidos no pronto atendimento da emergência do Hospital de Base. Arq Ciênc Saúde. 2007;14(4):193-7.

23. Korhonen N, Niemi S, Palvanem M, Parkkari J, Sievanen H, Kannus P. Declining age-adjusted incidence of fall-induced injuries among elderly finns. Age Ageing. 2012;41(1):75-9.

24. Lojudice DC, Laprega MR, Rodrigues RAP, Rodrigues Junior AL. Quedas de idosos institucionalizados: ocorrência e fatores associados. Rev Bras Geriatr Gerontol. 2010;13(3):403-12.

25. Siqueira FV, Facchini LA, Piccini RX, Tomasi E, Thumé E, Silveira DS. Prevalência de quedas em idosos e fatores associados. Rev Saúde Pública. 2007;41(5):749-756.

26. Motta LB, Aguiar AC, Coutinho ESF, Huf G. Prevalência e fatores associados a quedas em idosos em um município do Rio de Janeiro. Rev Bras Geriatr Gerontol. 2010;13(1):83-92.

27. Camarano AA, Kanso S, Mello JL. Como vive o idoso brasileiro. In: Camarano AA. Os novos idosos brasileiros: muito além dos 60 ? Rio de Janeiro: Ipea; 2004. cap. 1. p. 26-73.

28. Instituto Brasileiro de Geografia e Estatística [Internet]. Síntese de Indicadores Sociais: uma análise das condições de vida da população brasileira 2010. Disponível em: http://www.ibge.gov.br. Acesso em: 9 dez. 2012.

29. Gawryszewski VP. A importância das quedas no mesmo nível entre idosos no estado de São Paulo. Rev Assoc Med Bras. 2010;56(2):162-7 
30. Pinto TCA, Maciel SML, Xavier AFC, Pinto AKA, Cavalcanti AL. Morbidade por causas externas em idosos e sua relação com lesões maxilofaciais. Pesq Bras Odontoped Clín Integr. 2008;8(2):159-64.

31. Ribeiro AQ, Rozenfeld $\mathrm{S}$, Klein $\mathrm{CH}$, César CC, Acurcio FA. Inquérito sobre uso de medicamentos por idosos aposentados, Belo Horizonte, MG. Rev Saúde Pública. 2008;42(4):724-32.

32. Paschoal SM, Salles RFN, Franco RP. Epidemiologia do Envelhecimento. $2^{\text {a }}$ ed. São Paulo: Atheneu; 2005.

33. Meireles VC, Matsuda LM, Coimbra JAH, Mathias TAF. Características dos idosos em área de abrangência do Programa Saúde da Família na região Nordeste do Paraná: contribuições para a gestão do cuidado em Enfermagem. Saúde Soc. 2007;16(1):69-80.

34. Gonçalves LG, Vieira ST, Siqueira FV, Hallal PC. Prevalência de quedas em idosos asilados do município de Rio Grande, RS. Rev Saúde Pública. 2008;42(5):938-45.
35. Antes DL. Quedas e fatores associados em idosos de Florianópolis SC: estudo EpiFloripa Idoso. Dissertação (Mestrado) Universidade Federal de Santa Catarina, Florianópolis, 2011.

36. Barbosa MLJ, Nascimento EFA. Incidência de internações de idosos por motivo de quedas em um hospital geral de Taubaté. Rev Biociênc. 2001;7(1):35-42.

37. Filgueiras MC, Santiago FR, Santiago HAR, Vieira LJES. Fraturas em idosos decorrentes de quedas registradas em hospital terciário de referência em traumatologia no ano de 2004. Rev Bras Promoç Saúde. 2007;20(4):226-32.

38. Coutinho ESF, Bloch KV, Coeli CM. Mortalidade em um ano de idosos após hospitalização por fratura decorrente de queda: comparação com idosos pareados da população. Cad Saúde Pública. 2012;28(4):801-5.

39. Rezende DAP, Pereira WMP, Schmitt ACB, Pereira ECA, Aldrigh JM. Prevalência de quedas em mulheres após menopausa. Rev Bras Crescimento Desenvolv Hum. 2011;21(1):146-55. 